\title{
Aortic Valve Replacement in Patients with Small Aortic Annulus: New Pericardial Stented Valves vs Aortic Root Enlargement Procedures.
}

\author{
Ahmed Fouad $^{1}$, ehab elshihy ${ }^{1}$, Mohammed Hassan ${ }^{1}$, Mohammed Maged ${ }^{1}$, and Ashraf \\ Mostafa Abd Raboh ${ }^{1}$ \\ ${ }^{1}$ Cairo University Kasr Alainy Faculty of Medicine
}

December 13, 2021

\begin{abstract}
Objectives: Newer generations of stented pericardial valves may offer hemodynamic benefit in patients with small aortic annulus. The aim of this study was to determine the effectiveness of isolated aortic valve replacement with one such valve, the Trifecta valve, when compared to Aortic root enlargement surgery in reducing postoperative gradients and the severity of PPM in patients with small aortic annulus. Patients and methods: A prospective observational study of 100 patients with SAA who underwent AVR from March 2020 to October 2021 in Cairo university hospitals and other centers. The cohort was divided into two groups based on surgical technique: Isolated AVR using Trifecta valve or ARE and mechanical valve placement. Preoperative characteristics, intraoperative times and postoperative outcomes were recorded and compared in all patients, including a pre-discharge echocardiography. Results: Increased operative times, increased ICU stay and need for blood products were observed in the ARE group and operative time was determined as an independent risk factor. Higher rate of complications such as need for permanent pacemaker as well increased postoperative drainage was also recorded in ARE group, with no difference between groups in in-hospital mortality. Higher incidence of PPM was recorded in the Trifecta group (24\%) compared to the ARE group (8\%). but, the severity of PPM within the Trifecta group was reduced compared to the ARE group, and no degree of PPM was observed in Trifecta valves sized 21. Conclusion The Trifecta valve offers excellent postoperative hemodynamics and significant reduction in severity of PPM in patients with SAA undergoing AVR, with gradients and iEOA almost comparable to larger sized valves implanted after ARE, making the increased surgical burden of ARE unnecessary in most patients.
\end{abstract}

\section{Hosted file}

AVR vs ARE.docx available at https://authorea.com/users/450981/articles/549242-aortic-valvereplacement-in-patients-with-small-aortic-annulus-new-pericardial-stented-valves-vsaortic-root-enlargement-procedures 